\title{
The Study on the Cycloids of Moving Loops
}

\author{
Gennady Tarabrin \\ Department of Applied Mathematics, Volgograd State Technical University, Volgograd, Russia \\ Email: tageti@mail.ru
}

How to cite this paper: Tarabrin, G. (2018) The Study on the Cycloids of Moving Loops. Journal of Applied Mathematics and Physics, 6, 817-830. https://doi.org/10.4236/jamp.2018.64070

Received: March 5, 2018

Accepted: April 21, 2018

Published: April 24, 2018

Copyright $\odot 2018$ by author and Scientific Research Publishing Inc. This work is licensed under the Creative Commons Attribution International License (CC BY 4.0).

http://creativecommons.org/licenses/by/4.0/

\begin{abstract}
The infinite set of cycloids is created. Each cycloid of this set is defined as a movement trajectory of a point when this point circulates on the convex closed contour of arbitrary form when this contour moves rectilinearly without rotation on the plane with a velocity equal to the tangential velocity of a point on circulation contour. The classical cycloid is elements of this set. The differential equation of a cycloid set is derived and its solution in quadratures is received. The inverse problem when for the given cycloid it is necessary to fine the form of a circulation contour is solved. The problem of differential equation of the second order with boundary conditions about a bend of big curvature of an elastic rod of infinite length is solved in quadratures. Geometry of the loop which is formed at such bend is investigated. It is discovered that at movement of an elastic loop on a rod when the form and the size of a loop don't change, each point of a loop moves on a trajectory which named by us the cycloid and which represents a circumference arch.
\end{abstract}

\section{Keywords}

Curves, Loops, Cycloids

\section{Introduction}

The work stated below is not generalization by someone before the executed researches and not development by someone before the offered methods. It is completely original work. Together with it, this work isn't beyond real activity of the person and consequently it bases on all fundamental knowledge saved up by people. The content of this work was published in Russian [1] [2] [3].

This paper contains two independent, but connected with each other, parts. These parts are united that both they can be classified as the problems of differential geometry about infinite curves with a loop which is formed by self-crossing of branches of these curves. Difference of parts is caused by the loops investigated below are made of materials of essentially different properties. 
In the first part, the loop is formed by a flexible string. Cross section of such string hasn't the effort named bending moment. Ideal variant such string is a continuous chain formed from rings.

In the second part, the loop is formed by ideally elastic rod. By example of such is a loop made from a steel string of a guitar. In cross section of such rod at its bend, there is bending moment.

In both parts of this work, according to the author conception, investigation of the loops form is not the main of article sense. The loops form is studied in combination with movement of these loops. Such statement of a problem results in kinematics research of loops points. The loops kinematics solves problems about the form of movement trajectories of loops points. The geometrical sense of these trajectories is similar to sense of a classical cycloid, therefore, in paper the points trajectories of loops we called cycloids.

Both parts of this paper investigate geometrical and kinematic properties of abstract objects. However, we will find out easily in them an opportunity of practical applications, for example, for track of the transport machines, for tractors, for military equipment, for the problems solving of formation prevention of hoses loops of divers and astronauts and etc.

\section{Cycloids of Circulation on Moving Contours}

The mechanical model which we will create represents mechanical system which points move on the cycloid studied by us. As a result we will receive mechanical system which scheme is represented on Figure 1. For creation of model we will perform the following operations: take inextensible ideally flexible string of infinitely big length, take absolutely rigid rod which cross section is limited to the closed convex contour, wrap up string once around a rod, and stretch this string by efforts $P$ made on infinity. Therefore, the string forms a flat loop that all its points without exception coincide with points of the edge of the rod cross section. The intersection point of the loop branches lays on the contour, and at the same time this point lays on a straight line along which the string is stretched by efforts $P$. This straight line represents the loop branches with endings remote in infinity. We will imagine that the contour (it is the edge of cross section of absolutely rigid rod) moves without rotation on a straight line coincident with stretched string. In the course of the contour movement the string which wrap round this contour is sliding on the contour. We will note by color tag a point on one of loop branches in the intersection point of loop branches. It will be that point which will begin to move on the contour. The color tag will make a circulation (it will make a full turnaround of contour) and stop movement in the intersection point of the loop branches. The color tag during of circulation will pass the distance is equal to length of all loop. At the same time the intersection point of the loop branches will go this distance on the straight line along which the string is stretched. The describe movement of the color tag on the rod at forward movement of this rod represents mechanical model of circulation on a moving contour. 


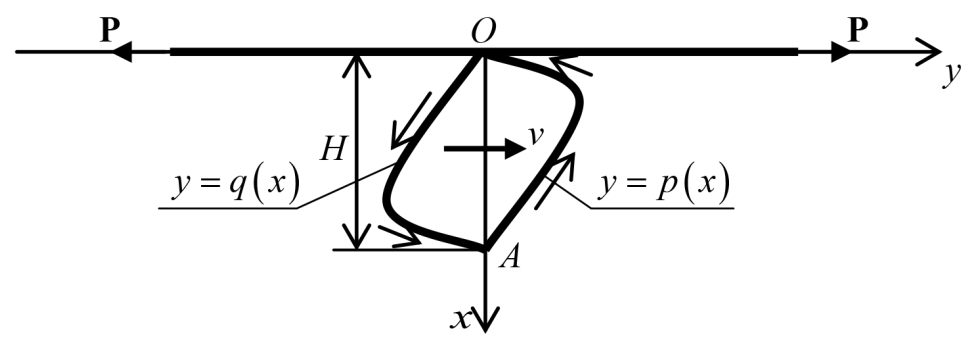

Figure 1. The schematic view of circulation on a moving contour.

We will place mechanical model in rectangular Cartesian coordinates $x, y$ so that the stretched string coincided with $y$-axis and that the loop thus is disposed on non-negative half of $x$-axis (Figure 1). We will suppose that at the supervision beginning moment over contour movement the intersection point of the loop branches was located into the coordinate's origin $O$. Not changing the form, the contour goes without rotation on a straight line aside a positive direction of $y$-axis. As the string all time remains stretched the form of string loop will remain constant, and the intersection point of the string branches at sliding a string on a contour during a contour movement will move on $y$-axis.

We will discriminate the string branches: the right-hand branch is curved in the positive side of $y$-axis, its curvilinear part is disposed on a positive half of $y$-axis and its rectilinear part is disposed on a negative half of $y$-axis; the left-hand branch is curved in the negative side of $y$-axis, its curvilinear part is disposed on a negative half of $y$-axis and its rectilinear part is disposed on a positive half of $y$-axis. On Figure 1 we see: point $O$ is branches intersection point, point $A$ is join point of the right-hand and left-hand branches. Point $A$ is disposed on a positive half of $X$-axis.

Let $H$ be a distance between points $O$ and $A$.

We will designate differently the equations of the right-hand and left-hand curvilinear parts of loop. Let at the observation beginning moment of contour movement an equality $y=p(x)$ be the equation of the right-hand loop branch and $y=q(x)$ be the equation of the left-hand loop branch. Then:

$p(x)>0, q(x)<0$ when $0<x<H$ and $p(0)=p(H)=q(0)=q(H)=0$.

Let's mark by marker a point on a string on its rectilinear site of the left-hand branch. We will denote this point by letter $M$. The contour goes to the right. Thus the intersection point of the string (loop) branches comes nearer to $M$-point. At the observation beginning moment $M$-point coincides with the coordinate's origin $O(0,0)$. At this time moment $M$-point begins the movement on a contour counter-clockwise. We will name this movement the circulation. Simultaneously with it $M$-point will make movement aside a positive direction of $y$-axis. This movement is named the transportation motion. We will observe the $M$-point movement which will be superposition of its both movements (circulation and transportation motion), and we will name a trajectory of this movement (movements superposition) the cycloid of circulation on a moving contour. 
Mathematical methods of this article unit aren't beyond traditional sections of textbooks of differential and integral calculus [4].

Let $L$ be length of the contour left-hand part from $O$-point up to $A$-point. The contour goes rectilinearly to the positive side of $y$-axis. When the contour will pass the $L$-distance, the $M$-point will pass by the contour left-hand part from $O$-point up to $A$-point. Taking into account both movement kinds (circulation and transportation) the marked $M$-point on coordinate's plane $x, y$ will be in $A_{1}$ -point with coordinates $x=H, y=L$. Let $v$ be velocity of transportation motion of $M$-point. Movement velocity of $M$-point on an arch length of the contour, it is tangential velocity, also will be equal to $v$. This statement follows from the preservation law of a string quantity in an intersection point of the string branches: the string quantity which left the right-hand part of a contour equals the string quantity which entered into the left-hand part of a contour. Thus it is necessary to take into account that the intersection point of the string branches goes with velocity $v$.

Let $\boldsymbol{V}=V_{x} \boldsymbol{i}+V_{y} \boldsymbol{j}$ be a vector of resulting velocity of $M$-point which is equal to the vector sum of tangential and transportation velocities. Let $\alpha$ be an angle between a straight line tangent to the function graph $y=q(x)$ and the $x$-axis. Components $V_{x}, V_{y}$ of a velocity vector $\boldsymbol{V}=V_{x} \mathbf{i}+V_{y} \mathbf{j}$ of $M$-point are equal to

$$
\left.\begin{array}{l}
V_{x}=v \cos (\alpha), \\
V_{y}=v[1+\sin (\alpha)]
\end{array}\right\} \Rightarrow\left\{\begin{array}{l}
V_{x}=v\left[1+\tan ^{2}(\alpha)\right]^{-1 / 2}, \\
V_{y}=v\left\{1+\left[1+\tan ^{2}(\alpha)\right]^{-1 / 2} \tan (\alpha)\right\} .
\end{array}\right.
$$

According to geometrical sense of the first derivative function $y=q(x)$, we have $\tan \alpha=q^{\prime}(x)$. Taking into account it we derive

$$
V_{x}=v\left(1+\left[q^{\prime}(x)\right]^{2}\right)^{-1 / 2}, \quad V_{y}=v\left\{1+q^{\prime}(x)\left(1+\left[q^{\prime}(x)\right]^{2}\right)^{-1 / 2}\right\} \text {. }
$$

Let $y=c(x)$ be the equation of a $M$-point movement trajectory and let $\beta$ be an angle between a tangent to the diagram of this trajectory and $x$-axis. According to geometrical sense of derivative function $y=c(x)$, we have $c^{\prime}(x)=\tan (\beta)$. According to physical sense of a vector $\boldsymbol{V}=V_{x} \boldsymbol{i}+V_{y} \boldsymbol{j}$, it is directed on a tangent to a $M$-point movement trajectory. Therefore $V_{x} / V_{y}=\tan (\beta)=c^{\prime}(x)$. Substituting here formulas $V_{x}, V_{y}$ we will derive the differential equation of a $M$-point movement trajectory

$$
c^{\prime}(x)=q^{\prime}(x)+\sqrt{1+\left[q^{\prime}(x)\right]^{2}} .
$$

Integrating this equation with the initial condition $c(0)=0$, we derive the equation of the left-hand half of the cycloid generated by the $M$-point movement on the left-hand branch of a loop

$$
y=c(x) \equiv q(x)+\int_{0}^{x} \sqrt{1+\left[q^{\prime}(\xi)\right]^{2}} \mathrm{~d} \xi, \quad 0 \leq x \leq H .
$$

In this equation the integral with a variable top limit determines the current 
value of an arch length of the left-hand half of a contour (the current value of distance which $M$-point passed on an arch of the left-hand half of a loop)

$$
l(x)=\int_{0}^{x} \sqrt{1+\left[q^{\prime}(\xi)\right]^{2}} \mathrm{~d} \xi .
$$

The formula (2) can be written down in a form

$$
y=q(x)+l(x) .
$$

This finite equation analytically defines any of a cycloid of an infinite set the elements of which were defined above by mechanical model. This result can be presented by the Theorem. At movement of a circulation contour in a positive direction of an ordinates axis at a abscissa growth stage of the cycloid current point the ordinate of the cycloid current point is equal to the sum of two components: the current point ordinate of a circulation contour and the current arch length of the passable contour part.

Symmetrical cycloids are result of circulation on symmetric contours. The symmetry axis is a straight line which is a perpendicular to a straight line in which direction the contour moves. The left-hand and right-hand branches of such contours submit to equality $p(x)=-q(x)$.

Let $L$ be a length of the left-hand loop branch. Then

$$
L=\int_{0}^{H} \sqrt{1+\left[q^{\prime}(\xi)\right]^{2}} \mathrm{~d} \xi .
$$

The Equation (4) describes a cycloid for $y \in[0, L]$. Then the equation of a symmetric cycloid for $y \in[L, 2 L]$ is expressed by the formula

$$
y=2 L-[q(x)+l(x)] .
$$

We will consider some special cases of a symmetric cycloid.

Circular (classical) cycloid (Figure 2 ) is result of circulation on a circumference. Let $R$ be a circumference radius. Then $(x-R)^{2}+y^{2}=R^{2}$ is the equation of a circulation contour with $H=2 R$. On formulas (2), (3) we derive

$$
\begin{gathered}
y=p(x)=-q(x) \equiv R \sqrt{1-(x / R-1)^{2}}, \quad 0 \leq x \leq 2 R, \\
l(x)=R[\pi / 2+\arcsin (x / R-1)] .
\end{gathered}
$$

Calculations on formulas (4), (5) give

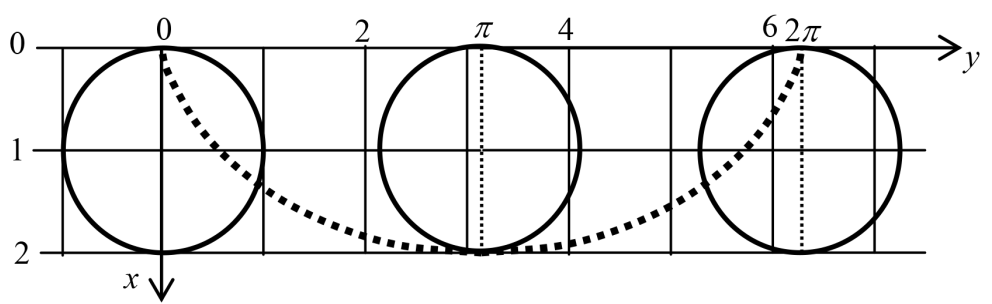

Figure 2. Circular (classical) cycloid-dashed line, $R=1$. 


$$
\begin{gathered}
y \in[0, \pi R] \quad y=R\left[\pi / 2+\arcsin (x / R-1)-\sqrt{1-(x / R-1)^{2}}\right], \quad 0 \leq x \leq 2 R ; \\
y \in[\pi R, 2 \pi R] \quad y=R\left[3 \pi / 2-\arcsin (x / R-1)+\sqrt{1-(x / R-1)^{2}}\right], \quad 0 \leq x \leq 2 R .
\end{gathered}
$$

Traditionally classical cycloid is defined by system of the parametrical equations. In our case the cycloid is unrolled along $y$-axis. Therefore in our case the classical cycloid is defined by the equations

$$
x=R(1-\cos t), \quad y=R(t-\sin t) .
$$

Substitution of these formulas in the first equation of a cycloid written down above gives identity. The identity result means that the deduced formula sets the left-hand half of a classical cycloid. After that the equation of the right-hand half of cycloid does not cause doubts.

Parabolic cycloid (Figure 3 ) is a result of a point circulation on a moving contour which is formed by the functions graphs

$$
y=p(x)=-q(x) \equiv 4 h(x / H)(1-x / H), \quad 0 \leq x \leq H,
$$

where $h$-height of a parabolic arch.

Current arch length such contour according to formula (3) is defined on formula

$$
l(x)=\int_{0}^{x} \sqrt{1+(4 h / H)^{2}(1-2 \xi / H)^{2}} \mathrm{~d} \xi .
$$

On a formula (4) the diagram of a parabolic cycloid has been constructed.

Elliptic cycloid (Figure 4 ) is a result of a point circulation on a moving contour in form of an ellipse. Let $a, b$ be ellipse semi axis. Then $(x-a)^{2} / a^{2}+y^{2} / b^{2}=1$ is the circulation contour equation with $H=2 a$ and on formula (2) we derive

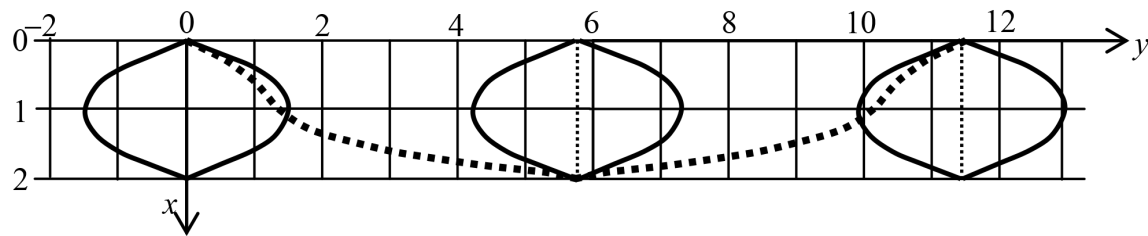

Figure 3. Parabolic cycloid-dashed line, $H=2, h=1.5$.

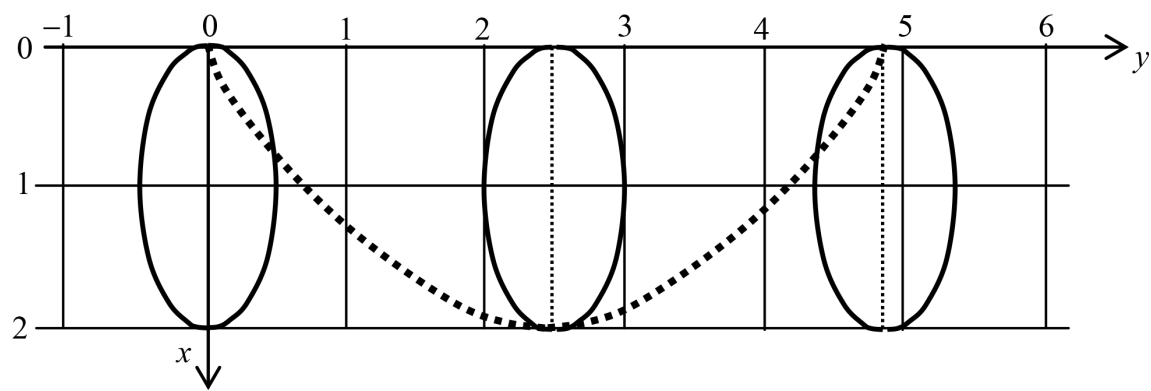

Figure 4. Elliptic cycloid-dashed line, $a=1, b=0.5$. 


$$
y=p(x)=-q(x) \equiv b \sqrt{1-(x / a-1)^{2}}, \quad 0 \leq x \leq 2 a .
$$

Calculation on formula (3) reduces to an elliptical integral

$$
l(x)=\int_{0}^{x} \sqrt{\left\{1-\left[1-(b / a)^{2}\right](\xi / a-1)^{2}\right\}\left[1-(\xi / a-1)^{2}\right]^{-1}} \mathrm{~d} \xi, \quad 0 \leq x \leq 2 a .
$$

On a formula (4) the diagram of a elliptic cycloid has been constructed.

Non-symmetrical cycloids are generated by circulation on non-symmetrical contours. Lack of a contour symmetry should be relative to a straight line which is perpendicular to the direction of the contour movement and means that the inequality $p(x) \neq-q(x)$ is carried out. For $y \in[0, L]$ non-symmetrical cycloid equation is described as in symmetrical case by the formula (4). The second part of the non-symmetrical cycloid is described by equation

$$
y=\int_{0}^{H} \sqrt{1+\left[q^{\prime}(\xi)\right]^{2}} \mathrm{~d} \xi+p(x)+\int_{x}^{H} \sqrt{1+\left[p^{\prime}(\xi)\right]^{2}} \mathrm{~d} \xi .
$$

Inverse problem about cycloid of circulation on a moving contour appears if in Equation (1) functions $q(x)$ and $c(x)$ exchange roles with each other. Solving the Equation (1) with unknown $q^{\prime}(x)$ and integrating $q^{\prime}(x)$, we derive

$$
q(x)=\frac{1}{2}\left[c(x)-\int \frac{\mathrm{d} x}{c^{\prime}(x)}\right]+C,
$$

where $C$ is arbitrary constant. This formula gives the reason to pose a question about the solution of an inverse problem about cycloid of circulation on a moving contour: to derive the equation of a moving contour from the given a cycloid equation. The formula (6) defines the equation of the left-hand part of a circulation contour within arbitrary constant $C$-summand. To find unknown (required to us) the contour equation, it is necessary to calculate the value of $C$-constant. The cycloid is a trajectory of the movement of the marked point which circulates on a contour and a contour at the same time moves rectilinearly without rotation. So any beginning of movement of the marked point is a point of a cycloid origin and is also a point of circulation origin on a contour. Consequently in the inverse problem considered here the coordinates of a point of a cycloid origin should be given together with the cycloid equation. If in the equation (6) to make substitution $c(x)$ and to execute integration we will derive a formula of the curves set for the given cycloid. This set contains required curve. If after that to replace variables $x, y$ by the point coordinates of a cycloid origin then we will derive the equation the solution of which will give us the $C$-constant value corresponding to the given cycloid. Thus the inverse problem will be solved and this solution will be only one.

For example, let the symmetric cycloid is given by function

$$
c(x)= \begin{cases}\left(\sqrt{a^{2}+b^{2}}-b\right) x / a & \text { if } 0 \leq x \leq a, \\ \left(\sqrt{a^{2}+b^{2}}+b\right) x / a-2 b & \text { if } a<x \leq 2 a .\end{cases}
$$


Here the cycloid equation is given and simultaneously with it the origin point $(0,0)$ of the curve is given. Calculation by the formula (6) gives formula

$$
y=p(x)=-q(x) \equiv \begin{cases}b x / a & \text { if } 0 \leq x \leq a, \\ 2 b-b x / a & \text { if } a<x \leq 2 a .\end{cases}
$$

This formula determines a contour in the form of a rhombus with semi-axes $a, b$. On Figure 5 the given cycloid and a rhombus inducing it are showed, where $a=1.5, b=1$.

\section{Loop on Elastic Infinite Rod}

Statement of elastic loop problem. The elastic rod of infinite length is stretched by forces $P$ which are applied on indefinitely far the rod ends. On the rod there is an elastic loop. This loop is formed by single fold self-crossing of two half of the considered rod. The elastic loop was studied by L. Euler [5]. But it was the loop which was formed at longitudinal compression of a rod of finite length. This loop is named Euler's elastic.

We will place the rod with the loop in rectangular Cartesian coordinates $x, y$ so that the rod will settled down along $y$-axis and the loop was directed to the positive side of $x$-axis. Thus $y$-axis will be the curve asymptote representing the rod with the loop. Symmetry axis of the loop we will combine with $y$-axis.

We will discriminate two loop branches: the right-hand branch which is curved in the positive side of $y$-axis (its left end is stretched in negative infinity), and the left branch which is curved in the negative side of $x$-axis (its right end is stretched in positive infinity). On Figure 6 we see: $O$-coordinate's origin, $A$-branches intersection point of the loop, $B$-join point of the right-hand and left-hand of the loop branches. Let $h$ be a distance between $O$-point and $A$-point, we will name $h$ the intersection point level; let $H$ be a distance between $O$-point and $B$-point, we will name $H$ the loop height.

Let $y=e(x)$ be an equation of right-hand loop branch. Then $e(x)<0$ when $0<x<h, e(x)>0$ when $h<x<H, e(h)=e(H)=0, \lim _{x \rightarrow 0} e(x)=-\infty$.

We will assume that during formation of a loop the rod was bent under laws of deformation of an elastic thin beam. Let $E$ be Young's modulus of a rod material.

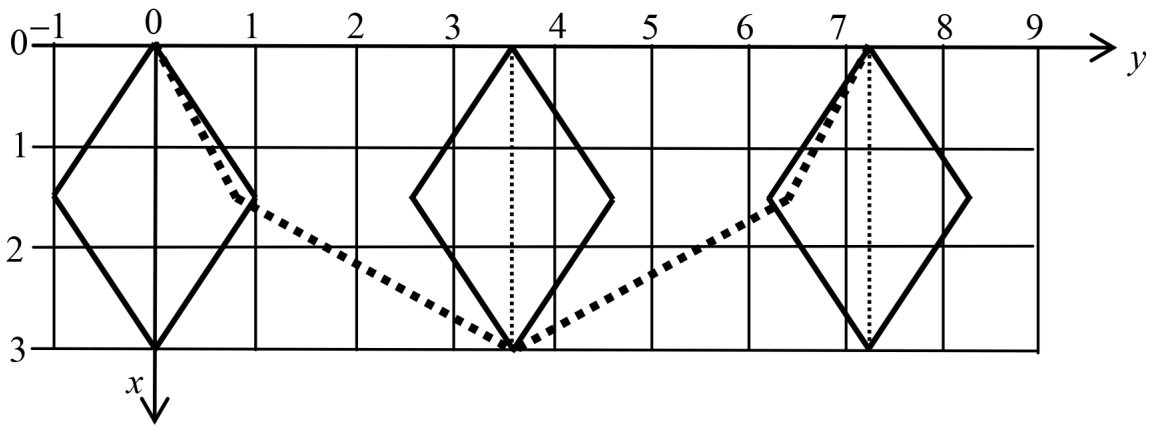

Figure 5. Rhombic cycloid-dashed line. 


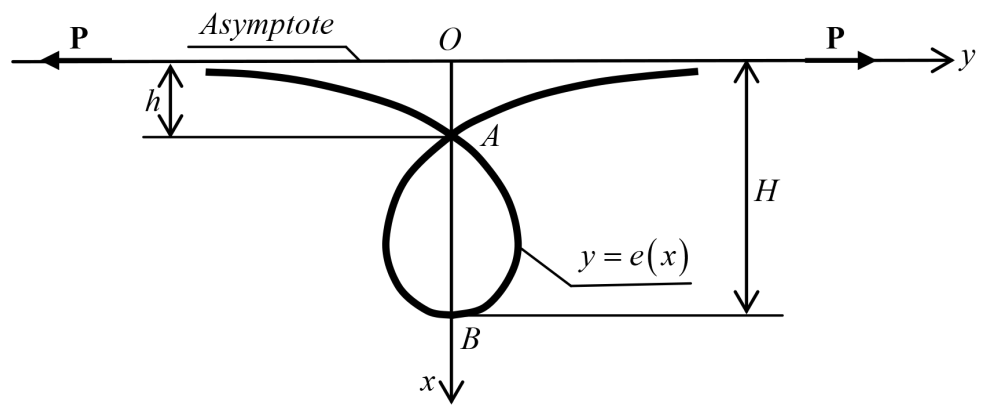

Figure 6. Loop on elastic infinite rod.

The rod which we study is a thin beam which bend theory is created with use of the simplifying Bernoulli's hypotheses [6]:

1) Hypothesis about flat sections: a cross section which is flat at the not deformed beam remains flat during a bend and makes thus rotate around of a neutral axis;

2) Hypothesis about the linear stress condition: normal stress $\sigma$ and relative linear strain $\varepsilon$ of longitudinal fibers of a rod are interlinked by Hook's law $\sigma=\varepsilon E$;

3) Hypothesis about even distribution: rod fibers which are equidistant from a neutral axis have equal stresses.

We will designate a symbol $I$ the inertia axial moment of a rod cross section in a bend plane.

Equation of the elastic loop form. Curve curvature in the current point of the bent rod axis within the framework of Bernoulli's hypotheses is directly proportional to the bending moment which is acting in cross section of a rod of this axis point. For the right-hand branch this statement is expressed by equality

$$
e^{\prime \prime}(x)\left\{1+\left[e^{\prime}(x)\right]^{2}\right\}^{-3 / 2}=-P x / E I,
$$

which is the differential equation of the elastic loop form.

We will enter a new variable $z(x)=e^{\prime}(x)$ therefore the equation (7) will be transformed to the equation of the first order

$$
z^{\prime}(x)\left[1+z^{2}(x)\right]^{-3 / 2}=-P x / E I .
$$

We will make replacement of a variable $z(x)=\sinh [t(x)]$ and we will integrate. The common solution of this equation is

$$
e^{\prime}(x)\left\{1+\left[e^{\prime}(x)\right]^{2}\right\}^{-1 / 2}=-P x^{2} / 2 E I+C,
$$

where $C$ is arbitrary constant.

On Figure 6 we see, that on right-hand branch $e^{\prime}(x) \rightarrow+\infty$ when $x \rightarrow 0+0$. We subordinate the Equation (8) this condition and in result we derive $C=1$.

The elastic rod with a loop which we examine is everywhere the smooth figure symmetric relative to $x$-axis. Therefore on right-hand branch $e^{\prime}(x) \rightarrow-\infty$ 
when $x \rightarrow H-0$. We subordinate the equation (8) with $C=1$ this condition and in result we derive

$$
P=4 E I / H^{2} .
$$

We will substitute $C=1$ and $P=4 E I / H^{2}$ into the equation (8) and solve this equation about unknown $e^{\prime}(x)$. In result we will have

$$
e^{\prime}(x)=\left[2(x / H) \sqrt{1-(x / H)^{2}}\right]^{-1}-(x / H)\left[1-(x / H)^{2}\right]^{-1 / 2}, \quad 0<x<H .
$$

We will make substitution $t=\sqrt{1-(x / H)^{2}}$. It will allow us to have

$$
\int\left[(x / H) \sqrt{1-(x / H)^{2}}\right]^{-1} \mathrm{~d} x=-H \ln \left\{\left[1+\sqrt{1-(x / H)^{2}}\right] /(x / H)\right\} .
$$

We shall make substitution $t=1-(x / H)^{2}$. It will allow us to have

$$
\int(x / H)\left[1-(x / H)^{2}\right]^{-1 / 2} \mathrm{~d} x=-H \sqrt{1-(x / H)^{2}} .
$$

After that we will find uncertain integral from function (8). We will take a constant of integration such that condition $e(H)=0$ was satisfied. It will be function which will represent the finite equation of the right-hand part of an elastic rod axis with a loop

$$
y=e(x) \equiv H\left\{\sqrt{1-(x / H)^{2}}-\frac{1}{2} \ln \left[\frac{1+\sqrt{1-(x / H)^{2}}}{x / H}\right]\right\}, \quad 0<x<H .
$$

Obviously, the point ordinates of the left-hand loop branch will have opposite signs.

Geometrical characteristics of elastic loop. We will construct a loop-1 by height $H_{1}=H$. The equation of this loop will be (11). Let's take on a loop-1 any way two points $M_{1}\left(a_{1}, b_{1}\right), M_{2}\left(a_{2}, b_{2}\right)$. At substitution of points coordinates $M_{1}, M_{2}$ into Equation (11) we will derive two the identities which will have of a form

$$
b=H\left\{\sqrt{1-(a / H)^{2}}-\frac{1}{2} \ln \left[\left(1+\sqrt{1-(a / H)^{2}}\right) /(a / H)\right]\right\} .
$$

Let's construct different loop it will be a loop-2 by height $H_{2}=k H$ where $k=$ const $>0$. The equation of this loop will be

$$
y=e(x) \equiv k H\left\{\sqrt{1-(x / k H)^{2}}-\frac{1}{2} \ln \left[\frac{1+\sqrt{1-(x / k H)^{2}}}{x / k H}\right]\right\}, \quad 0<x<k H .
$$

We will take two points $N_{1}\left(k a_{1}, k b_{1}\right), N_{2}\left(k a_{2}, k b_{2}\right)$. At substitution of points coordinates $N_{1}, N_{2}$ into Equation (13) we will derive two the identities which will have a form also (12). Hence points $N_{1}, N_{2}$ lay on the loop-2.

Now we will calculate distances between points on both loops

$$
M_{1} M_{2}=\sqrt{\left(a_{2}-a_{1}\right)^{2}+\left(b_{2}-b_{1}\right)^{2}},
$$




$$
N_{1} N_{2}=\sqrt{\left(k a_{2}-k a_{1}\right)^{2}+\left(k b_{2}-k b_{1}\right)^{2}}=k \sqrt{\left(a_{2}-a_{1}\right)^{2}+\left(b_{2}-b_{1}\right)^{2}} .
$$

As a result of comparison of these distances we derive

$$
N_{1} N_{2} / M_{1} M_{2}=k=H_{2} / H_{1} \text {. }
$$

This equality means, that the loop-1 and a loop- 2 are geometrically similar figures, their similarity coefficient is equal to $k=H_{2} / H_{1}$. This fact gives the reason to formulate the following fundamental characteristics of loops on elastic infinite rods.

Theorem. All loops on elastic infinite rods are in pairs the geometrically similar figures with the similarity coefficient equal to the ratio of their heights.

Similarity of loops opens an opportunity to deduce formulas of geometrical forms and these formulas will be suitable for rods of any source data values $P, E, I$.

We have by formula (9)

$$
H=2 \sqrt{E I / P}
$$

We will talk the unitary loop if $H=1$. It can be at $P=4 E I$.

The branches intersection point lies on $x$-axis. Therefore $e(h)=0$. Substituting $h=\eta H$ into the formula (11), we derive

$$
\sqrt{1-\eta^{2}}=(1 / 2) \ln \left[\left(1+\sqrt{1-\eta^{2}}\right) / \eta\right] \text {. }
$$

We will solve this equation. In result of it we will have

$$
\eta \approx 0.288 \text {. }
$$

$\eta$-number is the level of the branches intersection point of the unitary loop. We will name $\eta$-number the unitary level.

The current length of a loop arch with the origin in the intersection point $A$ is determined by the formula

$$
l(x)=\int_{h}^{x} \sqrt{1+\left[e^{\prime}(x)\right]^{2}} \mathrm{~d} x, \quad h \leq x \leq H .
$$

Substituting here formula (10) and calculating quadratures we derive

$$
l(x)=H\left\{\pi_{e}-(1 / 2) \ln \left[\left(1+\sqrt{1-(x / H)^{2}}\right) /(x / H)\right]\right\}, \quad h \leq x \leq H,
$$

where $\pi_{e}$ is some constant. We will calculate it.

As the origin point of the current length of a loop arch is in the intersection point $A$, then $l(h)=0$. We will substitute $h=\eta H$ into the formula (15) and will use the formula (14). In result from the equation $l(h)=0$ we will derive

$$
\begin{gathered}
\pi_{e}-(1 / 2) \ln \left[\left(1+\sqrt{1-\eta^{2}}\right) / \eta\right]=0 \Rightarrow \pi_{e}-\sqrt{1-\eta^{2}}=0 \Rightarrow \\
\pi_{e}^{2}+\eta^{2}=1 .
\end{gathered}
$$

As we already calculated $\eta$-number, from this equation we determine

$$
\pi_{e} \approx 0.958 \text {. }
$$


By formula (15)

$$
L=l(H)=\pi_{e} H \quad \Rightarrow \quad \pi_{e}=L / H .
$$

Number $L$ is equal to a half loop length. Consequently $\pi_{e}$-number is the quotient of a half loop length to height of this loop. If $H=1$, then $\pi_{e}$-number is half length of unitary loop. $\pi_{e}$-number we will name the $\pi$-number of an elastic loop.

The loop branches are crossing with angle $\gamma$. Making use the formula (10) at $x=\eta H \quad$ we derive

$$
\gamma=2 \arctan \left[e^{\prime}(\eta H)\right] \Rightarrow \gamma \approx 0.628 \pi \sim 113^{\circ} .
$$

Numbers $\eta, \pi_{e}, \gamma$ are absolutely constants of the elastic loops on infinity rod.

The Equation (16) is the geometrical identity of an elastic loop.

The current area $s(x)$ between a loop branches when $x \geq h$ is defined by formula

$$
\begin{gathered}
s(x)=2 \int_{h}^{x} e(\xi) \mathrm{d} \xi \Rightarrow \\
s(x)=\left\{\eta \pi_{e}+\frac{x}{H}\left[\sqrt{1-\left(\frac{x}{H}\right)^{2}}-\ln \frac{1+\sqrt{1-(x / H)^{2}}}{x / H}\right]\right\} H^{2}, \quad h \leq x \leq H .
\end{gathered}
$$

From here the area of an elastic loop is equal to

$$
S=\eta \pi_{e} H^{2} .
$$

Cycloid of elastic loop. The infinite elastic rod with loop is mechanical system. The loop of this system can move forward without the form change on a straight line parallel to the loop asymptote (parallel to $y$-axis). In practice it can be observed, for example, on air hoses for divers. Some of physicists are inclined to believe that loop movement on infinite elastic rod is a soliton (solitary wave) [7].

We will consider that the loop goes to the positive side of $y$-axis. On a rod on the positive side of $y$-axis we will mark $M$-point. When the branches intersection point will come in $M$-point, the $M$-point will start movement on a loop. This movement of the marked point on a loop will repeat all the movement laws which we considered above in section "Circulation cycloids on moving contours". Therefore now we have the right to take results of that section and we make use of that.

Into the cycloid Equation (4) we will make the following two replacements: the left-hand half of an elastic loop $y=q(x) \equiv-e(x)$ in the form (11) and the current arch length $l(x)$ of an elastic loop in the form (15). As a result we will derive the equation of the first half of the circulation cycloid on an elastic loop

$$
y=H\left[\pi_{e}-\sqrt{1-(x / H)^{2}}\right], \quad h \leq x \leq H .
$$


The considered cycloid is symmetric. Therefore from the formula (5) we derive the equation of the cycloid second half of an elastic loop

$$
y=H\left[\pi_{e}+\sqrt{1-(x / H)^{2}}\right], \quad h \leq x \leq H .
$$

We will transform the Equations (17), (18) to the form

$$
(x-0)^{2}+\left(y-\pi_{e} H\right)^{2}=H^{2} .
$$

This equation is the canonical circumference equation of radius $H$ with the circumference centre into a point with coordinates $x=0, y=\pi_{e} H$.

The cycloid origin is the point $(h, 0)$, the cycloid ending is the point $\left(h, 2 \pi_{e} H\right)$.

In Figure 7 there is the elastic loop cycloid (dotted graph) and three loop positions (firm lines) which we have made by formulas (11), (17), (18).

\section{Conclusions}

Geometry of movement trajectory of a point at its circulation on rectilinearly moving contour is investigated. We have named such trajectories cycloids by analogy to a classical cycloid which is a point trajectory of a circumference when the circumference rolls on a straight line without sliding. On kinematics of mechanical model of circulation on a moving contour, we have formulated the general definition of all sets of considered cycloids. We have derived the differential equations of these cycloids and have solved in quadratures the boundary problems of the derived equations. Cycloids curves for particular forms of circulation contours are constructed. We have solved the inverse cycloid problem of point circulation on a moving contour. This is the problem about finding of a circulation contour for given cycloid equation.

Results of research of an elastic loop have practical significance as the loops on the elastic infinite rods excite special attention in many technological processes and technical decisions.

It is established that an elastic loop on an infinite rod possesses marvelous geometrical properties. To feel it, we compare an elastic loop to a circumference.

All loops on the elastic infinite rods by pairs are the geometrically similar figures with the similarity coefficients which are equal to the quotient of their heights $H$.

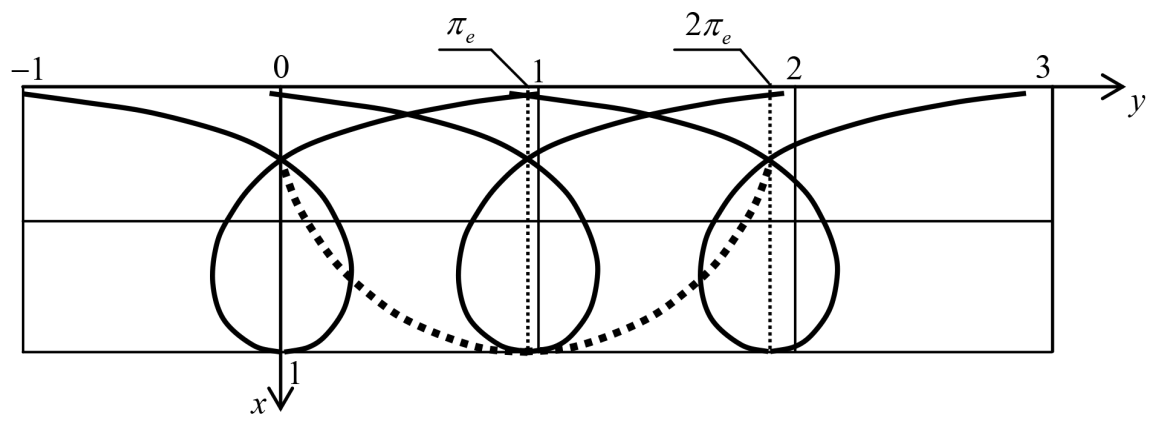

Figure 7. Cycloid of elastic loop. 
All circumferences by pairs are the geometrically similar figures with the similarity coefficients which are equal to the quotient of their radii $R$.

Half length: of a loop equals to $L=\pi_{e} H$, of a circumference equals to $L=\pi R$.

Area: of a loop equals to $S=\eta \pi_{e} H^{2}$, of a circle equals to $S=\pi R^{2}$.

Arch of a circumference is a cycloid of a loop on the elastic infinite rod.

The result of comparison between a loop and circumference gives the basis to name a loop on an elastic infinite rod, the sibling of a circumference.

\section{References}

[1] Tarabrin, G.T. (2012) Loop on Elastic Rod. Stroitelnaya Mechanika i Raschet Sooruzheniy, 3, 34-39. (In Russian)

[2] Tarabrin, G.T. (2012) Circulations Cycloids on Moving Counters. Stroitelnaya Mechanika i Raschet Sooruzheniy, 4, 38-43. (In Russian)

[3] Tarabrin, G. (2014) Non-Chrestomathy Problems of Mathematical Physics. Palmarium Academic Publishing, Saarbrucken. (In Russian)

[4] Piskunov, N. (1975) Differential and Integral Calculus. Vol. 1, Mir Publishers, Moscow.

[5] Rabotnov, J.N. (1988) Mechanics of Deformable Solid. Nauka, Moscow. (In Russian)

[6] Timoshenko, S. (1955) Strength of Materials. D. van Nostrand Company, Inc., Princeton, New Jersey, Toronto, New York, London.

[7] Filippov, A.T. (1990) Multiform Soliton. Nauka, Moscow. (In Russian). 